\title{
Public acceptability of government interventions to reduce obesity: policy effectiveness, policy fairness, government trust and political ideology
}

\author{
Anoma Gunarathne, Achim Spiller and Antje Risius \\ Department of Agricultural Economics and Rural Development, Marketing for Food and Agricultural Products, \\ University of Goettingen, Goettingen, Germany
}

\section{Abstract}

Frequent consumption of sugar-sweetened beverages (SSBs) has been linked to a greater risk of obesity, type 2 diabetes mellitus, dental caries and coronary heart disease. SSBs are an ideal target for public health intervention to help control the type 2 diabetes mellitus epidemics since they have absolutely no nutritional value, and do not protect against disease. However, successful implementation of policies to reduce consumption of SSBs depends on public acceptability and support. Therefore, determining the public acceptability of government interventions to reduce sugar-sweetened beverages is critical for effectiveness as well as implementation. The aim of the present study was to conduct an empirical investigation of how different policy-related factors (namely perceived policy effectiveness, perceived policy fairness, policy trust and political ideology) link to public support. Policy options included low, medium and high intensity versions of: imposing a tax $(10 \%, 20 \%$ and 30\%); adding a warning label (with and without graphic warning label); reducing number of SSB retail outlets (schools only and all public institutions); and regulating SSB advertising (ban advertising aimed at children and ban all SSB advertising). Data were collected from a representative sample of 948 consumers (age range from 18 to 69 ) through an online survey, which was carried out in February 2019. Sample quotas were set for age, gender and income. The partial least squares approach of the structural equation modelling (PLS-SEM) is employed to investigate direct effects of the proposed factors on public acceptance. Importantly, the results indicate that perceived fairness and perceived effectiveness of the political interventions had a significantly positive correlation with public acceptance $(\beta=0.518$, $p$-value $<0.001$ and $\beta=0.299$, p-value $<0.001$, respectively). Meanwhile, those who have a greater trust in government are more likely to support $(\beta=0.049$, $p$-value $<0.05)$. Also, public acceptance is positively associated with liberal policy strains. This is in line with social cognitive motives such as openness to new experiences and desire for equality in society. Moreover, higher trust in government is positively associated with perceived fairness as well as perceived effectiveness. These findings reflect that those who trust government believe that the government policy is fair in terms of the policy itself, how it is established as well as its specific distributive effects. Therefore, participatory policymaking that involves the public in the decision-making process can enhance citizens' trust and overall acceptance in policy interventions with regard to SSBs.

\section{Conflict of Interest}

There is no conflict of interest 